\title{
Needle Chlorophyll Content Estimation: A Comparative Study of PROSPECT and LIBERTY
}

\author{
Inian Moorthy ${ }^{1}$, John R. Miller ${ }^{2}$, Pablo J. Zarco-Tejada ${ }^{3}$, and Thomas L. Noland ${ }^{4}$ \\ ${ }^{1}$ Centre for Research in Earth and Space Science (CRESS), York University, Toronto, Canada - moorthy@terra.phys.yorku.ca \\ 2 Department of Physics and Astronomy, York University, Toronto, Canada. \\ ${ }^{3}$ Grupo de Óptica Atmosférica (GOA-UVA), Universidad de Valladolid, Spain. \\ ${ }^{4}$ Ontario Forest Research Institute (OFRI), Ministry of Natural Resources, Sault Ste. Marie, Canada.
}

\begin{abstract}
Inversion capabilities of leaf-level radiative transfer models, PROSPECT and LIBERTY, were evaluated to estimate needle chlorophyll content from measured needle reflectance and transmittance spectra. Sensitivity studies, using simulation results, were conducted on the models' biophysical parameters to determine robust inversion approaches. Unlike PROSPECT, which has only one scattering parameter $(N)$, the LIBERTY model is comprised of two highly sensitive scattering parameters (average cell diameter and intercellular air gap), which influence visible and near infrared spectral characteristics and challenge usual iterative minimization techniques. Validation of inversion algorithms were based on Jack Pine (Pinus Banksiana) needles, collected in forested areas near Sudbury, Ontario, Canada, between June and September of 2001 for laboratory spectral and biochemical measurements. Both PROSPECT and LIBERTY were capable of accurately inverting model-simulated reflectance and transmittance spectra for needle pigment content. However, inversions using actual data have proven more challenging. For PROSPECT, with an empirically determined geometric form factor to account for needle shape, inversions using measured spectra yield pigment estimates with an RMSE of $13 \mathrm{ug} / \mathrm{cm}^{2}$ over a pigment range between 15 and $68 \mathrm{ug} / \mathrm{cm}^{2}$. For LIBERTY, current efforts focus on effectively accounting for the highly sensitive model scattering parameters to permit accurate inversion of measured needle spectra for pigment estimation. Comparisons of inversion results using both PROSPECT and LIBERTY, as well as contrasts in the sensitivity of specific model parameters to retrieval accuracy are presented. Such assessments of leaf-model inversion capabilities are necessary prior to coupling it with canopy models to make stand level pigment estimations.
\end{abstract}

Keywords - Chlorophyll, PROSPECT, LIBERTY, Radiative Tranfer Models, Model Inversion

\section{INTRODUCTION}

Understanding the optical properties of vegetation canopies, first requires a knowledge of the spectral characteristics (reflectance, transmission, and absorption) of individual leaves. Concentrations of different biochemical constituents, namely, pigments, water, cellulose, lignin, and nitrogen, control absorption features evident in leaf reflectance spectra. Absorption characteristics in the visible domain $(400-700 \mathrm{~nm})$ are due to electron energy transitions in chlorophyll and carotenoid pigments [1]. In the near infrared region $(1000-2500 \mathrm{~nm})$, absorption features are a function of bending and stretching vibrations of bonds between hydrogen-carbon and nitrogen-oxygen atoms [2]. Based on these absorption properties, measurements of leaf reflectance, at fine spectral resolution, can be used to estimate relative concentrations of the different biochemical constituents. These leaf-level estimates can then be used in conjunction with canopy-level models to develop "bioindicators" of vegetation stress and photosynthetic efficiency at local to regional scales.

There are generally two approaches in determining the relationship between biochemical components and foliar optical properties. Firstly, statistical associations between leaf reflectance/transmittance and biochemical concentration, and secondly, physically based radiative transfer models of photon interaction with leaf absorption/scattering constituents. Although statistical approaches can establish important correlations, they offer no predictive capabilities and cannot be easily extrapolated to other species with different cell structures. The physical modeling approach, on the other hand, can provide accurate descriptions of the photon interaction with different leaf biochemical constituents and be potentially inverted for estimates of foliar biochemical content.

Currently, most leaf-level radiative transfer models are based upon broadleaf vegetation, where the anatomical leaf structure is defined by a series of layers with different plate/air space configurations. One of the most widely validated plate model to date is PROSPECT [3], which describes leaf optical properties from 400 to $2500 \mathrm{~nm}$ as a function of three parameters: a structure parameter $N$, chlorophyll concentration, and water content. Modeling approaches for coniferous needles, however, have been inhibited due to the problems of needle shape, thickness and internal cell structure. However, the recent introduction of the LIBERTY (Leaf Incorporating Biochemistry Exhibiting Reflectance and Transmittance Yields) model [4], allows the characterization of conifer needles, at the cellular scale, using Melamed's radiative transfer theory for suspended powders [5]. LIBERTY assumes that the needle structure is composed of roughly spherical cells, which are separated by air spaces. Initial inversion studies of simulated data demonstrated the potential of LIBERTY to estimate biochemical concentrations through minimization techniques. The 
inversion of LIBERTY, however, has to be accurately validated with real measurements of needle reflectance and transmittance.

\section{EXPERIMENTAL MATERIALS AND METHODS}

\section{A. LIBERTY Model Sensitivity}

The LIBERTY model employs 9 biophysical/chemical properties to simulate needle reflectance and transmittance between 400 and $2500 \mathrm{~nm}$ (Table I). The model linearly sums specific absorption coefficients that were measured during the BOREAS campaigns, scaled by their respective concentrations, to calculate a global absorption coefficient $K$. This global absorption coefficient, in conjunction with the structural parameters of average cell diameter, intercellular air gap and needle thickness are used to calculate needle reflectance and transmittance between 400 and $2500 \mathrm{~nm}$.

Since the focus of this investigation was to evaluate LIBERTY's potential to retrieve chlorophyll content, it was possible to reduce the number of parameters in question. Only parameters that contribute to the variation in needle reflectance between 400 and $800 \mathrm{~nm}$ were incorporated into this study. The effects of water, lignin/cellulose, and protein, on the modeled reflectance occur in the near infrared region at wavelengths greater than $1000 \mathrm{~nm}$ [4]. As a result, these parameters were held constant, with nominal values, and would not impact forward simulations or inversion studies. The remaining six parameters were varied between their lower and upper limits and the associated parameter sensitivity changes in simulated reflectance were observed.

TABLE I. LIBERTY INPUT PARAMETERS

\begin{tabular}{|c|c|c|}
\hline \multirow{2}{*}{ LIBERTY Parameter } & \multicolumn{2}{|c|}{ Parameter Characteristics } \\
\hline & Units & Range \\
\hline Average Cell Diameter $(D)$ & $\mu \mathrm{m}$ & $30-100$ \\
\hline Intercellular Air Space $(x u)$ & I & $0.01-0.10$ \\
\hline Baseline Absorption & l & $0.004-0.010$ \\
\hline Albino Absorption & I & $1-10$ \\
\hline Needle Thickness & 1 & $1-10$ \\
\hline Chlorophyll Concentration & $\mathrm{mg} / \mathrm{m}^{2}$ & $0-600$ \\
\hline Water Concentration & $\mathrm{g} / \mathrm{m}^{2}$ & $0-500$ \\
\hline Lignin/Cellulose Concentration & $\mathrm{g} / \mathrm{m}^{2}$ & $10-80$ \\
\hline Nitrogen Concentration & $\mathrm{g} / \mathrm{m}^{2}$ & $0.3-2.0$ \\
\hline
\end{tabular}

\section{B. LIBERTY Model Inversion}

The Newton Raphson iterative method was applied to the LIBERTY model to retrieve an inverted global absorption coefficient $K_{i n v}$ as a function of wavelength. Then, an unmixing algorithm was developed to separate the contribution of chlorophyll concentration to $K_{i n v}$, between 400 and $800 \mathrm{~nm}$, using an iterative optimization technique. In this experiment, a standard root mean square error (RMSE) was minimized to determine the optimum set of fitted parameters.
Model inversion ability decreases with increasing number of parameters. As a result, the LIBERTY model requires some prior knowledge of needle characteristics to invert reflectance values. More specifically, the inverse mode of the model requires user input of the average cell diameter $D$ and the intercellular air space $x u$. In this experiment, the inversion method was tested on simulated needle reflectance for chlorophyll content estimation as a function of prior knowledge of $D$ and $x u$. LIBERTY spectra were generated for varying chlorophyll concentration $\left(10\right.$ to $\left.600 \mathrm{mg} / \mathrm{m}^{2}\right)$. The chlorophyll retrieval accuracies were measured as function of user knowledge of $D$ and $x u$. With prior knowledge of both $D$ and $x u$, the algorithm retrieves the exact solution for chlorophyll content. If knowledge of both $D$ and $x u$ are unknown, the algorithm inverted for an estimation of $D$ and chlorophyll content using different guesses at $x u$. The sensitivities of these scattering parameters during model inversion were recorded for simulated spectra and its implications on real needle spectral measurements are discussed.

\section{Needle Reflectance/Transmittance Data}

In this experiment, the validation data comprised of foliage samples that were collected from eight selected Jack Pine (Pinus banksiana) sites near Sudbury, Ontario during August of 2001. Foliage from the upper canopy (exposed to sunlight) was acquired from five representative trees within each site. The samples were harvested using a shotgun and were temporarily stored in coolers during transport to the field laboratory, where reflectance and transmittance measurements were acquired using an Analytical Spectral Devices Full Range Spectroradiometer coupled with a LiCor 1800 integrating sphere. A black anodized carrier that presents only the needle surfaces to the integrating sphere was used to systematically measure the spectral properties of the Jack Pine foliage [6]. Although the use of the needle carrier eliminates the need for gap fraction estimators, it introduces the need to quantitatively compensate the effect of the carrier, in the reflectance and transmittance measurements. These corrections were carefully accounted for, and the resultant validation data were used in this experiment to assess the potential of inverting LIBERTY for pigment estimations. After the necessary spectral measurements were recorded, the samples were stored in a freezer for laboratory chlorophyll retrieval.

\section{EXPERIMENTAL RESULTS AND DISCUSSION}

The inversion algorithm, using 60 simulated spectra with a chlorophyll content range between 10 and $600 \mathrm{mg} / \mathrm{m}^{2}$, yielded a retrieval accuracy of $64.4 \mathrm{mg} / \mathrm{m}^{2}\left(\mathrm{r}^{2}=0.87\right)$, assuming no prior knowledge of $D$ and $x u$ (Figure 1). The LIBERTY model's inversion capabilities for chlorophyll content estimation are highly sensitive to the scattering parameters of $D$ and $x u$ (Figure 2).

Inversions of measured Jack Pine needle spectra revealed that the PROSPECT model yielded better retrievals then the LIBERTY model (Figure 3). PROPSECT was used because it is less complex then other leaf models, and is easily invertible due to its small number of input parameters. 


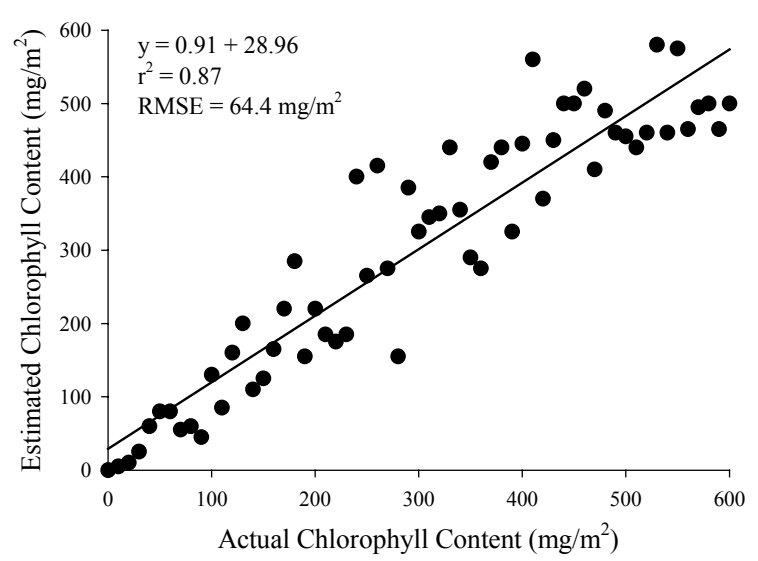

Figure 1. LIBERTY model inversions for chlorophyll content, using simlated spectra with no prior knowledge of $D$ and $x u$

Furthermore, PROSPECT forward simulations showed good agreement with needle measured spectra especially in the visible region, thereby offering the potential to be adapted to conifer needle cellular structures [7]. In this investigation, the PROSPECT model was adapted to the non-flat conifer needles, with the use of an empirically determined geometric form factor as measured from needle photomicrographs. The RMSE for the PROSPECT using 64 measured spectra was $12.7 \mathrm{ug} / \mathrm{cm}^{2}$, while LIBERTY produced an error of 22.9 $\mathrm{ug} / \mathrm{cm}^{2}$. There are several potential reasons for the LIBERTY's inability to properly invert the measured needle spectral measurements. Firstly, both the average cell diameter and the intercellular air space for the spectrally measured needles were unknown. Consequently, the inversion process can generate significant errors without such a-priori information. Next, another possible limitation includes the discrepancies between LIBERTY pigment absorption coefficients and the coefficients in PROSPECT. The local absorption maxima at $540 \mathrm{~nm}$ and $620 \mathrm{~nm}$ modeled in LIBERTY are not present in PROSPECT coefficients [4].
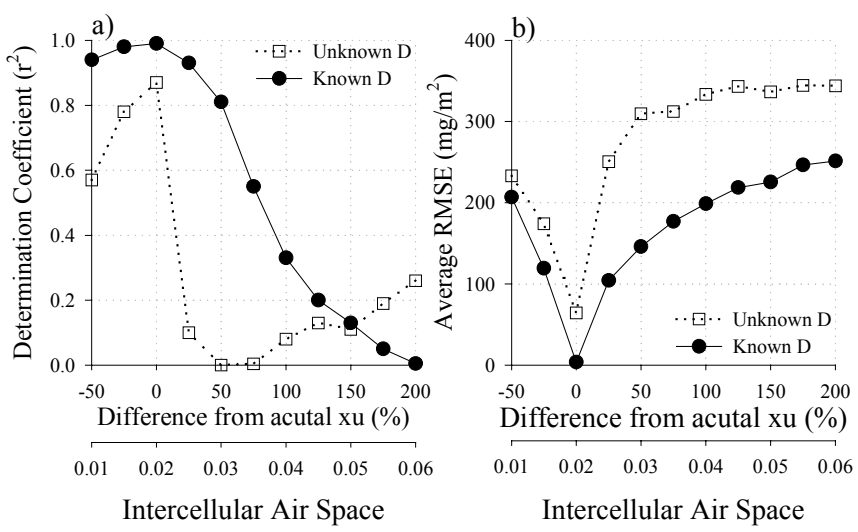

Figure 2. Variation in a) determination coefficient and b) RMSE as a function of prior knowledge of cell diameter $D$ and deviation from actual intercellular air space $x u$.

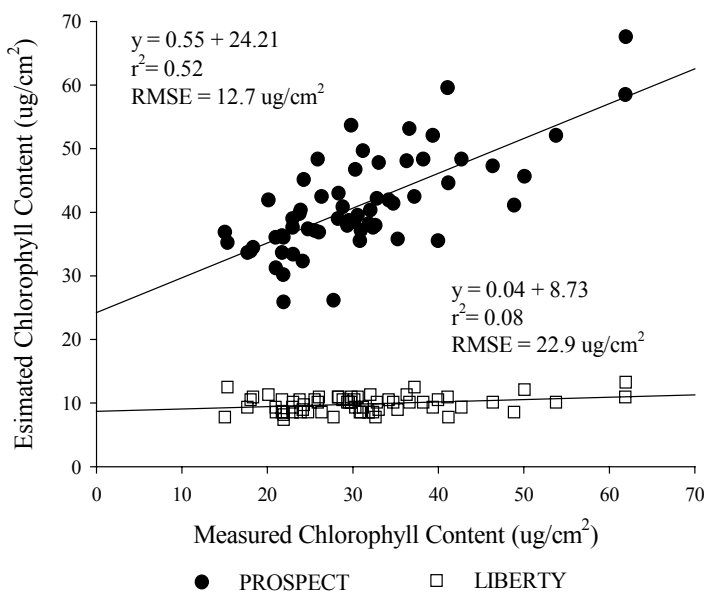

Figure 3. LIBERTY model inversions for chlorophyll content, using measured Jack Pine spectra with no prior knowledge of $D$ and $x u$.

In addition, there is a difference in the "green peak", local absorption minimum at $550 \mathrm{~nm}$ and $580 \mathrm{~nm}$ for PROSPECT and LIBERTY, respectively. These discrepancies, in conjunction with the lack of a-priori knowledge regarding the average cell diameter and intercellular air space have limited LIBERTY inversion capabilities.

\section{CONCLUSIONS}

This assessment of the LIBERTY model and its inversion capabilites highlights that the prior knowledge of two scattering parameters, namely the average cell diameter and the intercellular air space are critical to ensure acurate estimations of chlorophyll content. Current strategies to improve LIBERTY inversion include using photomicrographs of needle cross sections to mearsure $D$ and $x u$, and evaluating the imbedded variabilities in the absorption coefficients between PROSPECT and LIBERTY.

\section{REFERENCES}

[1] H.K. Lichtenhaler, "Chlorophylls and caratenoids: pigments of photosynthetic biomembranes," Methods Enzymol, vol. 148, pp. 350382, 1987.

[2] W. Kemp, Organic Spectroscopy. MacMillan, London, 1987.

[3] S. Jacquemoud, and F. Baret, "PROSPECT: A model of leaf optical properties," Remote Sensing of Environment, vol. 34, pp. 75-91, 1990.

[4] T.P. Dawson, P.J.Curran, and S.E. Plummer, "LIBERTY - Modeling the effets of leaf biochemical concentration on reflectance spectra," Remote Sensing of Environment, vol. 65, pp. 50-60, 1998.

[5] M.T. Melamed, "Optical properties of powders. Part I-Optical absorption coefficients and the absolute value ot the diffuse reflectance," Journal of Applied Physics, vol. 34, pp. 560-570, 1963.

[6] J. W. Harron "Optical properties of phytoelements in conifers," M.Sc. Thesis. Graduate Programme in Earth and Space Science, York University,Toronto, pp 193, 2000.

[7] P.J. Zarco-Tejada et al., "Needle chlorophyll content estimation through model inversion using hyperspectral data from boreal conifer forest canopies," Remote Sensing of Environment, in press. 